\title{
Laryngeal dystonia in the course of multiple system atrophy: a cause of postoperative respiratory insufficiency
}

\author{
Magdalena A. Wujtewicz $\cdot$ Kamil Chwojnicki • \\ Radosław Owczuk • Maria Wujtewicz
}

Received: 24 October 2010/ Accepted: 22 October 2011/Published online: 6 November 2011

(C) The Author(s) 2011. This article is published with open access at Springerlink.com

\begin{abstract}
Multiple system atrophy (MSA) is an adult onset, incurable neurodegenerative disease, characterized by symptoms of nervous system failure. Occurrence of laryngeal dystonia indicates increased risk of sudden death caused by airway occlusion. We present the case report of 63-year-old patient with history of orthostatic hypotension, parkinsonism, progressive adynamia, and stridor. The patient was admitted to the hospital for diagnosis of orthostatic hypotension. A diagnosis of possible MSA was made. Because of patient's complaints, an X-ray of the hip joint was taken. It revealed femoral neck fracture. Endoprosthesis insertion under general anesthesia was performed. Two days later the patient presented progressive adynamy and respiratory insufficiency. Endotracheal intubation and respiratory support were required followed by extubation and one more intubation. After second extubation, stridor and acute respiratory insufficiency occurred. Urgent tracheostomy was performed. After 13 days in ICU, the patient was discharged to the rehabilitation center.
\end{abstract}

Keywords Acute respiratory insufficiency · Multiple system atrophy $\cdot$ Laryngeal dystonia . Urgent tracheostomy

\section{Background}

Multiple system atrophy (MSA) is a heterogenic group of neurodegenerative diseases of the central and autonomic

M. A. Wujtewicz $(\bowtie) \cdot R$. Owczuk $\cdot$ M. Wujtewicz

Department of Anaesthesiology and Intensive Therapy,

Medical University of Gdańsk, Ul. Dębinki 7,

80-211 Gdańsk, Poland

e-mail: magwuj@gumed.edu.pl

K. Chwojnicki

Department of Neurology, Medical University of Gdańsk,

Gdańsk, Poland nervous systems. Clinical features include concomitant extrapyramidal disturbances, cerebellar and pyramidal symptoms, and dysautonomy. The prevalence of MSA is very low $(1.94$ to $4.4 / 100,000)$ with a typical age of onset in the 50 s. The incidence of disease is independent of gender [1,2]. Concerning neuropathology, MSA is an alpha-synucleinopathy [3]. Coexistence of Parkinson's symptoms with considerable autonomic dysfunction is referred to as Shy-Drager syndrome, after Dr. Milton Shy and Dr. Glenn Drager who identified it in 1960. However, it was redefined as MSA with autonomic phenomena in 1996 [4, 5]. Diagnosis of MSA is divided into the following three groups: definite MSA for subjects with autopsy confirmation, probable MSA for patients with autonomic failure and parkinsonism or cerebellar ataxia, and possible MSA for those in whom clinical findings do not yet clearly represent the disease [5].

In 2007, two clinical categories of MSA were distinguished according to their predominant symptoms: MSA-P predominated by Parkinson's syndrome and MSA-C with predomination of cerebellar disturbances [6]. Diagnosis in the living patient is based only on clinical features. Treatment is symptomatic and prognosis is detrimental. The lifespan following the first symptoms is usually no longer than 10 years [6].

\section{Case report}

We report the case of a patient with possible MSA who developed acute obstructive postoperative respiratory insufficiency caused by laryngeal dystonia. A 63-year-old female patient with a history of orthostatic hypotension, leading to collapses and loss of consciousness while tilting, was admitted to the medical ward of the university hospital for diagnosis. These progressive symptoms had been 
occurring for 5 years since 2005. During that time the patient's physical fitness had deteriorated; since 2008, she had been experiencing collapsing episodes. Neither tremors nor cognitive function disturbances were observed. The progressive loss of weight resulted the atrophy of all muscles. Several neurological consultations had been performed. Insufficiency of the suprarenal cortex had been excluded. The patient had been scheduled for magnetic resonance imaging (MRI), which would have been performed 5 weeks from the day of hospital admission after a general diagnostic process. Ten days before the planned hospital admission, the patient had fallen down once more. At that time, a neurologist made the diagnosis of Parkinson's disease and initiated treatment with L-Dopa (125 mg three times a day). However, there was no improvement. The general examination after admission to the hospital revealed orthostatic hypotension. No cardiologic disturbances were found. Neurological examination of the patient revealed: (1) symmetrical parkinsonian syndrome with bradykinesia and cogwheel rigidity, without resting tremor; (2) bilateral pyramidal signs-increased deep tendon reflexes and the Babinski sign; (3) well-expressed primitive (palmomental, snout, and glabellar) reflexes; and (4) mild intention tremor of the upper limbs.

The patient did not present any significant cognitive impairment (30 points on the Mini-Mental State Examination) [7] or oculomotor disorders. From anamnesis, it was found that the patient had been snoring for several weeks. Based on clinical findings, possible MSA, according to 2008 criterions was diagnosed [6]. Because of patient's complaints of leg pain, an X-ray of the hip joint was taken. This $\mathrm{X}$-ray revealed a femoral neck fracture with dislocation of the femoral shaft. The patient was then scheduled for insertion of a hip joint endoprosthesis. During the pre-anesthetic visit, she was adynamic and cardiovascularly stable; blood pressure (measured in the supine position) was 130/100 $\mathrm{mmHg}$; without stridor or signs of respiratory disturbances. The procedure was performed under general anesthesia (fentanyl, propofol, rocuronium, and a mixture of air and oxygen). Systolic blood pressure during anesthesia varied between 80 and $120 \mathrm{mmHg}$, and ephedrine was used to increase blood pressure. Profound hemodynamic instability was not observed. After the procedure, the patient was transferred to the surgical ward (bypassing the PACU) in good general condition.

Two days later, the patient started to demonstrate increasing adynamia. This adynamia led to respiratory distress with decreased percutaneous saturation to $90 \%$ despite oxygen supplementation. Desaturation continued and mental status worsened until the patient lost consciousness. After endotracheal intubation, the patient was admitted to the Intensive Care Unit (ICU). A short time later she regained consciousness; because of adynamia and visible respiratory efforts, however, assisted ventilation had to be continued for 2 days. An indirect laryngoscopy, performed after endotracheal tube removal, revealed the medium-wide glottis. The vocal cords were in the middle position and exhibited correct mobility. Twenty-seven hours later, loud stridor appeared and was followed by acute respiratory insufficiency. During an attack the patient presented stridor on inspiration, she was unable to speak.

The patient's trachea was intubated again for the next $34 \mathrm{~h}$. On the next day after tube removal, audible stridor reappeared. During urgent ENT consultation, neither disturbances of the vocal cords nor stridor was observed. However, there was marked retention of bronchial secretions. Bronchofiberoscopy was carried out, and considerably reduced mobility of the vocal cords was diagnosed. Subsequently, loud stridor and acute respiratory insufficiency occurred. A laryngeal mask airway was inserted, and percutaneous dilatation tracheostomy using the Griggs technique was immediately performed. Except for a short period right after this procedure, no more assisted ventilation was required. Suspicion of laryngeal dystonia was put forward. Concurrently, treatment with L-Dopa, previously withdrawn by the consulting neurologist, was restarted, and the dose was gradually increased to $4 \times 125 \mathrm{mg}$. The patient's condition stabilized. Thirteen days after admission to the ICU, the patient was transferred with good respiratory efficiency and a talking tracheostomy tube to a rehabilitation center. Diagnosis using central nervous system imaging (i.e., MRI) had to be postponed because of the recently implanted hip joint prosthesis. Consultation in an outpatient clinic for extrapyramidal diseases was recommended (in order to qualify the patient) for intralaryngeal injections with botulin toxin.

\section{Discussion}

The described case is an example of an interesting and very rare cause of acute respiratory insufficiency during the postoperative period-laryngeal dystonia in the course of MSA. Dystonia by itself is a frequent symptom of MSA. According to Boetsh, it occurs in the early stage of MSA in $46 \%$ of patients. The most frequent types are limb, cervical (in non-treated patients), and cranio-cervical (in patients treated with L-Dopa) dystonia [8]. Laryngeal dystonia is not a rare symptom, it can be found in $35-41 \%$ patients with MSA. It usually appears up to 3 years after the onset of disease and is rather typical for MSA-P. The occurrence of stridor is connected with poor prognosis-it indicates an increased risk of acute respiratory insufficiency and sudden death. The obstructive mechanism is dominating [9]. From her anamnesis, it was known that our patient has presented stridor for some period of time. 
The mechanism of laryngeal dystonia is connected with incorrect coactivation of the adductors and abductors of the vocal cords. Both palsy and hypertonicity of vocal cord muscles have been described [10, 11]. Stridor in MSA patients occurs predominantly during sleep [11]. Diagnosis of laryngeal dystonia has been indirectly confirmed in this case from the results of bronchofiberoscopic examination-reduced mobility of vocal cords.

Several methods, including plasty of the vocal cords, intralaryngeal injections with botulin toxin, and tracheostomy, are available to treat stridor in order to prevent acute respiratory distress. Additionally, the use of non-invasive ventilation with continuous positive airway pressure (CPAP) or bilevel positive airway pressure therapy while sleeping is used to prevent sudden nocturnal death [10]. With regard to cardiovascular instability, the drugs used most frequently to reduce orthostatic hypotension are midodrine and fludrocortisone [12].

Unfortunately, our patient was not treated with any of these methods preoperatively. Anesthesia in patients with MSA may be challenging. The type of anesthesia most frequently used in MSA patients is general, and this type was used in the present case. However, spinal anesthesia can also be suitable in such patients [13]. Dangerous airway obstruction upon induction of or emergence from general anesthesia may occur, but this obstruction is relatively easy to overcome by applying minimal positive pressure [14]. There were no such complications in this case. Intraoperatively, disturbances in the autonomic nervous system may result in defective baroreceptor reflexes and improper vasoconstriction. These problems might lead to an unpredictable reaction to the vasoconstrictors used to correct hypotension. No such response to ephedrine, used in the described case, was observed.

CPAP via a face mask may be beneficial in the treatment of stridor in MSA patients, and should be sufficient in the postoperative period to prevent airway occlusion [15]. The described patients had never been treated with CPAP. In cases such as this one, more aggressive procedures may be required. In this case, tracheostomy was performed as an urgency measure. Percutaneous dilatation tracheostomy using the Griggs technique was chosen because the anesthesiologist was very familiar with this technique, and the patient's neck anatomy was perfect for this type of procedure. The possibility of treatment with botulin injections in the near future was proposed to the patient at discharge.

\section{Conclusion}

The diagnosis of MSA is connected with the potential for various complications during the perioperative period. In particular, respiratory disturbances may be life threatening. Prevention of acute airway occlusion should be taken into account. Prolonged postoperative monitoring is required in patients with MSA, and this fact is confirmed by this case.

Open Access This article is distributed under the terms of the Creative Commons Attribution Noncommercial License which permits any noncommercial use, distribution, and reproduction in any medium, provided the original author(s) and source are credited.

\section{References}

1. Köllensperger M, Geser F, Seppi K, Stampfer-Kountchev M, Sawires M, Scherfler C, Boesch S, Mueller J, Koukouni V, Quinn N, Pellecchia MT, Barone P, Schimke N, Dodel R, Oertel W, Dupont E, Řstergaard K, Daniels C, Deuschl G, Gurevich T, Giladi N, Coelho M, Sampaio C, Nilsson C, Widner H, Del Sorbo F, Albanese A, Cardozo A, Tolosa E, Abele M, Klockgether T, Kamm C, Gasser T, Djaldetti R, Colosimo C, Meco G, Schrag A, Poewe W, Wenning GK (2008) Red flags for multiple system atrophy. Mov Disord 23:1093-1099

2. Wenning GK, Ben Shlomo Y, Magalhaes M, Daniel SE, Quinn NP (1994) Clinical features and natural history of multiple system atrophy: an analysis of 100 cases. Brain 117:835-845

3. Dickson DW, Lin W, Liu WK, Yen SH (1999) Multiple system atrophy: a sporadic synucleinopathy. Brain Pathol 9:721-732

4. Shy GM, Drager GA (1996) A neurological syndrome associated with orthostatic hypotension: a clinical-pathologic study. Arch Neurol 2:511-527

5. The Consensus Committee of the American Autonomic Society, The American Academy of Neurology (1996) Consensus statement on the definition of orthostatic hypotension, pure autonomic failure, multiple system atrophy. Neurology 46:1470

6. Gillman S, Wenning GK, Low PA, Brooks DJ, Mathias CJ, Trojanowski JQ, Wood NW, Colosimo C, Durr A, Fowler CJ, Kaufmann H, Klockgether T, Lees A, Poewe W, Quinn N, Revesz T, Robertson D, Sandroni P, Seppi K, Vidailhet M (2008) Second consensus statement on the diagnosis of multiple system atrophy. Neurology 71:670-676

7. Folstein MF, Folstein SE, McHugh PR (1975) Mini-mental state: a practical method for grading the cognitive stat of patients for the clinician. J Psychiatr Res 12:189-198

8. Boesch SM, Wenning GK, Ransmayr G, Poewe W (2002) Dystonia in multiple system atrophy. J Neurol Neurosurg Psychiatry 72:300-303

9. Munschauer FE, Loh L, Bannister R, Newsom-Davis J (1990) Abnormal respiration and sudden death during sleep in multiple system atrophy with autonomic failure. Neurology 40:677-679

10. Blumin JH, Berke GS (2002) Bilateral vocal fold paresis and multiple system atrophy. Arch Otolaryngol Head Neck Surg 128:1404-1407

11. Isozaki E, Shimizu T, Takamoto K, Horiguchi S, Hayashida T, Oda M, Tanabe H (1995) Vocal cord abductor paralysis (VCAP) in Parkinson's disease: difference from VCAP in multiple system atrophy. J Neurol Sci 130:197-202

12. Colosimo C, Pezzella FR (2002) The symptomatic treatment of multiple system atrophy. Eur J Neurol 9:195-199

13. Malinovsky JM, Cozian A, Rivault O (2003) Spinal anesthesia for transurethral prostatectomy in a patient with multiple system atrophy. Letter to the editor. Can J Anaesth 50:962-963

14. Lim YS, Kennedy NJ (2007) Multiple system atrophy as a cause of upper airway obstruction. Anesthesia 62:1179-1182

15. Kuźniar TJ, Morgenthaler TI, Prakash UB, Pallanch JF, Silber MH, Tippmann-Peikert M (2009) Effects of continuous positive airway pressure on stridor in multiple system atrophy-sleep laryngoscopy. J Clin Sleep Med 5:65-67 